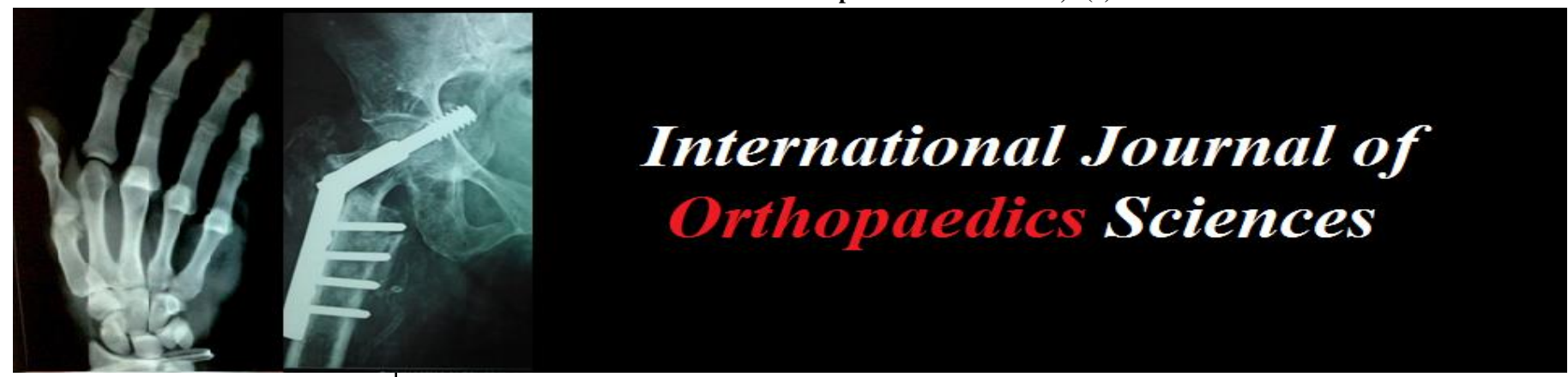

E-ISSN: 2395-1958

P-ISSN: 2706-6630

IJOS 2020; 6(2): 738-744

(C) 2020 IJOS

www.orthopaper.com

Received: 22-02-2020

Accepted: 24-03-2020

Dr. Nixon K Dias

Fellow in Arthroplasty

Hosmat Hospital 45, Magrath

Road, Richmond Road

Bengaluru, Karnataka, India

Dr. Deepak G Shivarathre

Consultant in Arthroplasty

Hosmat Hospital

Bangalore, Karnataka, India

Corresponding Author:

Dr. Nixon K Dia

Fellow in Arthroplasty

Hosmat Hospital 45, Magrath

Road, Richmond Road

Bengaluru, Karnataka, India

\section{A comparative study of patients undergoing total knee replacement with or without local corticosteroid injection for Pes anserine bursitis associated with osteoarthritis of the knee}

\author{
Dr. Nixon K Dias and Dr. Deepak G Shivarathre
}

DOI: https://doi.org/10.22271/ortho.2020.v6.i21.2133

Abstract

Pes anserine bursitis is one of the most important causes of periarticular pain. The prevalence of osteoarthritis together with Pes anserine bursitis has been reported to be as high as $75 \%$. Total knee replacement for knee osteoarthritis provides the greatest improvement in quality of life in patients with more severe functional impairment. The treatment options for pes anserine bursitis includes local injections of local anaesthetic (such as lidocaine) or a long acting corticosteroid. In our study we compared the functional outcomes in patients with osteoarthritis undergoing Total knee replacement along with or without local steroid injections for associated pes anserine bursitis. The objectives were to compare the pre and post operative pain levels with Visual Analogue Scale scores and functional outcomes with the Oxford knee scores in both these groups. All patients were divided into 2 groups "Yes" and "No", in a randomized fashion. The first group received a local injection at the site of the pes anserine bursitis along with total knee replacement surgery whereas the second group did not. It was observed that the pain scores in the "Yes" group, that received the local steroid injection were consistently lower than those in the "No" group, in the post operative follow up time intervals. However, this decrease in scores were not statistically significant. It was seen that both groups had a similar amount of significant improvement in functional scores at 1 month, and hence, the difference was insignificant. Local corticosteroid injections combined with Total knee replacement for pes anserine bursitis associated with osteoarthritis of the knee are safe but the efficacy of this treatment protocol could not be proved in our study. The results were not statistically significant probably due to the small sample size of the study.

Keywords: Osteoarthritis, Pes anserine bursitis, TKR, local corticosteroid

\section{Introduction}

Osteoarthritis of the knee is one of the most common forms of degenerative arthritis, and is a recurrent cause of chronic and severe knee pain in the elderly adult. The prevalence of this debilitating disease among the general population is around $10 \%$ to $12 \%$, thus making it one of the most common causes of pain, stiffness, limp and activity limitation ${ }^{[1]}$. Although synovitis is the causative pathology of an acute attack of osteoarthritis, it must be borne in mind that pes anserine bursitis (PAB) is commonly associated with osteoarthritis of the knee.

The term "pes anserinus" refers to the conjoined insertion of the sartorius, gracilis, and semitendinosus muscles along the proximal medial aspect of the tibia. These three muscles are primarily flexors of the knee; they also influence internal rotation of the tibia and protect the knee against rotatory and valgus stress. In pes anserine bursitis, the fluid-filled bursa in the conjoined insertion of the three hamstring muscles becomes inflamed and causes pain.

It has been estimated that $20 \%$ to $46 \%$ of the patients with symptomatic knee arthritis, have associated pes anserine syndrome ${ }^{2}$. Patients reporting a vague medial knee pain or with tenderness and swelling along the proximal medial tibia, may be easily misunderstood to be having an acute attack of osteoarthritis of the knee. Pes anserine syndrome is a clinical diagnosis with the predominant finding of tenderness over the proximal anteromedial tibia, approximately 2 to 3 inches below the knee. The exact source of the pain may originate from either the pes anserine tendons itself or the pes anserine bursa ${ }^{[3]}$. 
The symptoms of a pes anserine syndrome may be aggravated by ascending or descending stairs. The few conditions associated with this syndrome are degenerative osteoarthritis of knee, obesity, pes planus, etc.

The disease was described in 1973 by Brookler and Mongan, who reported to note the strong association between degenerative joint disease and the appearance of pes anserine bursitis. This study describes our experience with the epidemiology and treatment of this all too frequently associated syndrome. The prevalence of osteoarthritis together with PAB has been reported to be as high as $75 \%$.

There are various treatment modalities for this particular clinical picture. Treatment includes non-steroidal antiinflammatory drugs (NSAIDs), physiotherapy, and injections of corticosteroid. Responses may vary greatly between patients with recovery taking somewhere from 10 days to 36 months. Local injections of local anaesthetic (such as lidocaine) or a long acting corticosteroid is an effective and simple procedure.

Total knee replacement (TKR) for knee osteoarthritis provides the greatest improvement in quality of life in patients with more severe functional impairment, while the procedure produces only minimal improvement in patients with less severe impairment.

There have been studies which showed that patients with positive findings on an ultrasound examination, were the ones to effectively respond to a local corticosteroid injection ${ }^{4}$. An extra articular corticosteroid injection into the pes anserine bursa will need to be included with the treatment of the osteoarthritic knee, as this may equally limit the functional progression of the patient, just as the intra-articular pain. On many occasions, does a patient with these coexisting conditions return, complaining that the intra articular injection was not effective. However, on examination, it is revealed that the point source of tenderness is not in the joint line, but is located over the pes anserine bursa. Thus, awareness of this clinical picture, greatly assists them to fully participate in physical therapy.

The diagnosis is usually made on clinical grounds with tenderness located over the posteromedial aspect of the proximal tibia. Ultrasound, computed tomography and MRI are of limited value in diagnosing this condition. Uson et al. did not find any evidence of tendinitis or bursitis in patients diagnosed with pes anserine bursitis ${ }^{53}$. MRI can be useful in cases with acute pes anserine bursitis with fluid collection in the bursa. It can also be used to evaluate the other soft tissues around the knee at the time. The emphasis lies on the diagnosis and distinguishing this condition from osteoarthritis, as the treatment is fairly easy, with local injections of lidocaine and steroid providing good pain relief. In most cases, the occurrence of PAB in patients, cannot be proved; so the diagnosis of PAB syndrome is frequently based on clinical features. Ultrasonography can facilitate the diagnosis of PAB. However, in most suspected cases ultrasound findings are lacking. In a study, only $2.5 \%$ of these patients showed radiologic signs of $\mathrm{PAB}$ in magnetic resonance imaging (MRI) ${ }^{[6]}$; therefore the diagnosis of $\mathrm{PAB}$ cannot be based solely on MRI findings, but it is helpful for ruling out other structural causes of proximal tibial pain such as stress fracture and bone cysts.

Co-occurrence of periarticular soft tissue impairments may contribute to symptom severity, and may be associated with higher disability. More clinical studies, especially those using imaging techniques, are needed to establish the association between PAB, and symptom severity and disability in knee osteoarthritis.

Anesthetic (local) and corticosteroid combinations may be used. Local anesthetics stabilize neuronal membranes and prevent the initiation and transmission of nerve impulses, thereby producing local anesthesia. Corticosteroids have antiinflammatory properties and cause profound and varied metabolic effects; in addition, they modify the body's immune response to diverse stimuli.

\section{Materials and Methods \\ 2.1 Study Population}

The study was conducted at Hosmat Hospital, Bangalore after approval from the local ethics and advisory committee.

\subsection{Study Period}

From the period of July 2019 to December 2019 - 6 months

\subsection{Sample size}

A total of 24 patients and 30 knees were taken into study.

\subsection{Methodology}

All patients were diagnosed clinically, as having pes anserine bursitis in association with degenerative osteoarthritis of the knee. The clinical diagnosis was made by eliciting tenderness over the posteromedial aspect of the proximal tibia, below and away from the medial joint line, while placing the leg in a figure of four or externally rotated position, in a patient having coexisting degenerative osteoarthritis of the knee that was confirmed radiologically. On diagnosis of the pes anserine bursitis, the point of maximum tenderness was marked with a skin marker.

\subsubsection{Inclusion criteria}

- Patients with the diagnosis of Osteoarthritis knee with an associated pes anserine bursa tenderness, planning to undergo TKR.

- Age between 40 and 80 years.

- Any grade of degenerative arthritis of the knee

- Acute exacerbation of the pain of arthritis

- Patients with synovial inflammation

\subsubsection{Exclusion criteria}

- Patients with degenerative or non degenerative neurological diseases (change of patient's perception), history of coagulopathy, cancer, cardiac pacemaker, pregnancy, fractures below the knee during the past year, history of corticosteroid injection during the past month, knee effusion were not included.

- Hypersensitivity to any of the products

- Ongoing treatment with other immunosuppresants or corticosteroids

- Recent surgical intervention, 3 weeks before the study

- Significant co-morbidities like psychiatric disorders, immunodeficiency or uncontrolled diabetes, severe rheumatic disease.

The VAS score was taken by asking the patient to mark their level of pain on the scale from 0 (absence of pain) to 10 (unbearable pain). This score was taken before surgery along with the Oxford knee score that assessed pre operative functional limitations of the patients.

All patients who fitted the inclusion criteria, were divided into 2 groups "Yes" and "No" in a randomized fashion. The process of randomization was carried out by assigning patients to either group based upon the odd / even nature of 
their date of surgery.

The first group i.e. the test group, received a local injection at the site of the pes anserine tendinitis/bursitis along with total knee replacement surgery. The injection was given immediately after surgery as soon as closure of skin was complete. The mixture compromised of $1 \mathrm{ml}$ of local anaesthetic; lidocaine $2 \%$ and $1 \mathrm{ml}(40 \mathrm{mg})$ of the corticosteroid Triamcinolone.
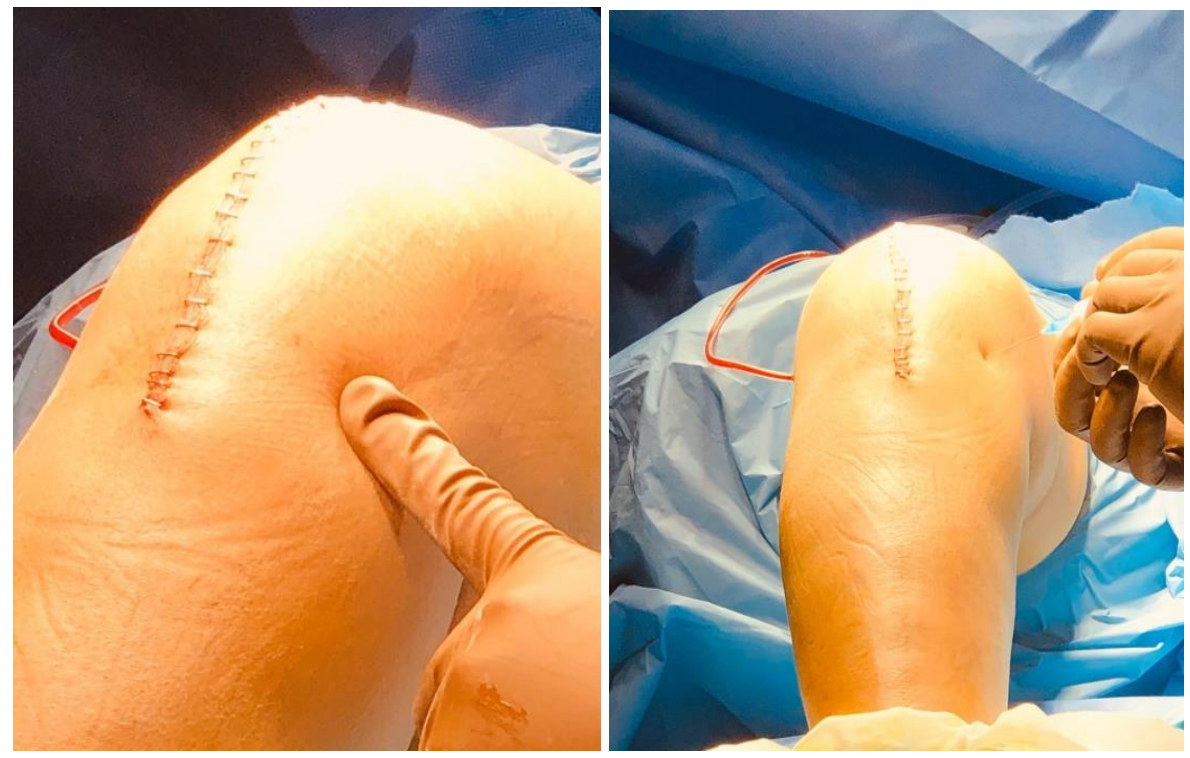

Point of local steroid injection

The second group, the control group, underwent the total knee replacement surgery but did not receive the local corticosteroid injection. After the dressing, compression bandage was applied along with a long knee brace, as was done for all operated knees.

Physiotherapy was commenced from the evening of the surgery, with calf and quadriceps strengthening exercises, as well as ROM. On the first post operative day, wound dressing was done and the drain was removed. A dressing was repeated on the $4^{\text {th }}$ post operative day, with a waterproof readymade dressing.

At the same time, on the $4^{\text {th }}$ postoperative day, the site of previous tenderness over the posteromedial tibia was reassessed. The VAS score was taken again and documented. The patient was discharged on regular medications, and athome physiotherapy was arranged.

\subsubsection{The discharge medications included}

- NSAIDs like Paracetamol or Aceclofenac for analgesia 1 week

- Pantoprazole for prevention of gastritis

- Ecospirin $75 \mathrm{mg}$ for 2 weeks for prevention of thrombotic disease post operatively

Patients were explained about the necessary precautions like avoiding cross legged sitting or squatting, and were advised full weight bearing mobilization with walker, and later, to transfer to a walking stick.

The patients were called back to the hospital for suture removal 14 days from the date of surgery. After wound inspection and suture removal, the VAS score was documented once again at 2 weeks post op. At the subsequent follow up, at 1 month, the VAS scores along with the Oxford Knee score were assessed once again.

\subsection{Statistical Analysis}

The data collected was entered into Microsoft excel spreadsheet and analyzed using IBM SPSS Statistics, Version 22(Armonk, NY: IBM Corp). Descriptive data were presented in the form of frequency, percentage for categorical variables and in the form of mean, median, standard deviation and quartiles for continuous variables. Comparisons of the categorical variables between the study groups were performed using the chi square test and fishers test. Variation in the study variables over different time periods were compared using repeated measures ANOVA with Bonferroni post hoc test. Comparison of the study variables between two groups at each time interval was performed using independent sample $t$ test. Comparison of oxford score between pre op and 1 month in each group was performed using paired t test. One way ANOVA was used to compare VAS and oxford scores, and according to BMI in each group. $\mathrm{P}$ value $<0.05$ was considered as statistically significant.

\section{Results}

In our study, it was observed that all patients that met the inclusion criteria were females. In fact, all patients that presented to our clinic with the syndrome of osteoarthritis with pes anserine bursitis, who were excluded from the study as per criteria, were also found to be female. Hence, the syndrome was observed to occur exclusively in females. The causes are yet to be understood.

Patients were divided into 4 categories on the basis of BMI. The categories were:

1. Normal $(18.5-24.9)$

2. Overweight $(25.0-29.9)$

3. Class I Obesity $(30.0-34.9)$

4. Class II Obesity $(35.0-39.9)$

None of the patients were in the underweight $(<18.5)$ or in the class iii (extreme) obesity category (>40.0). $23.3 \%$ of patients were in the normal category and $16.7 \%$ were in the overweight category. $30 \%$ of patients were in the class I obesity and class II obesity category respectively. on comparison between the study groups, there was no significant difference among the 4 categories, and hence they were comparable. Therefore, class I obesity and class II were the most common groups noted. 


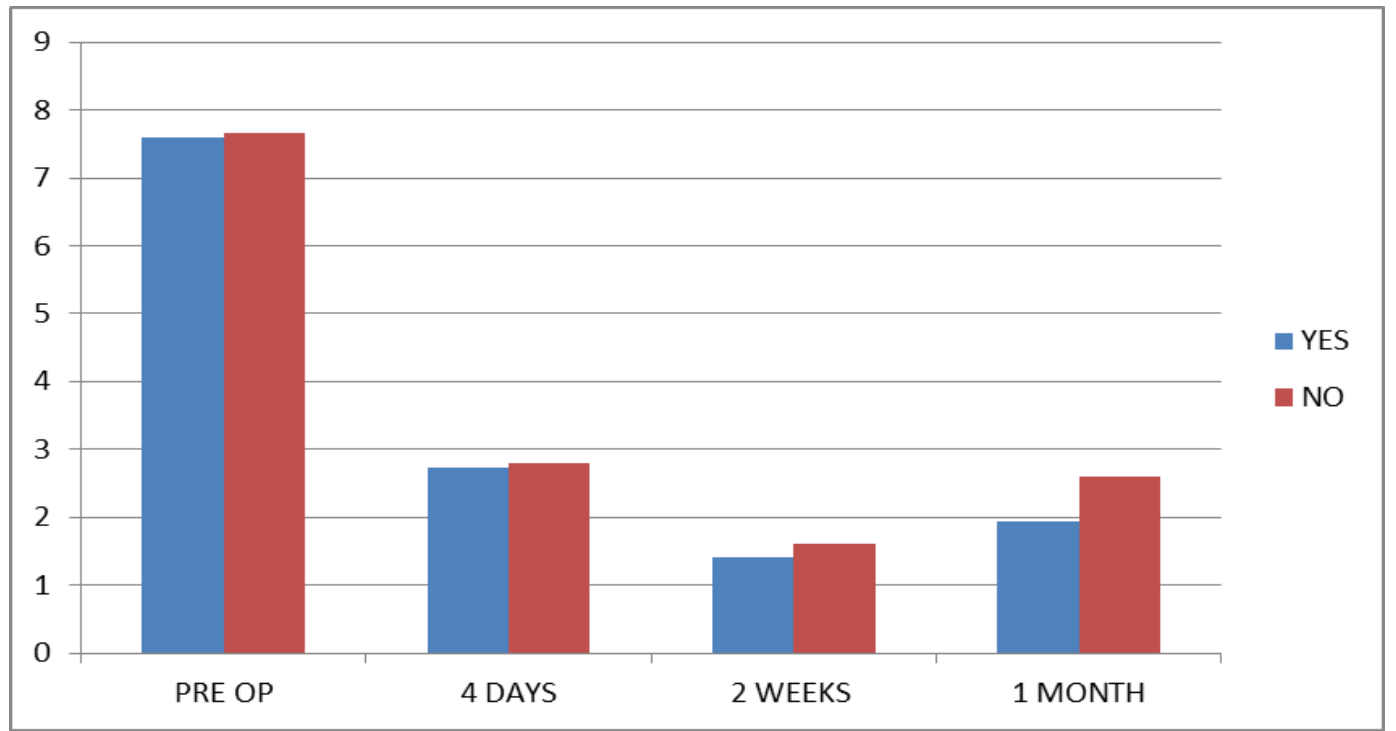

Graph 1: Comparison of VAS between the study groups at each time interval

From the above graph, it can be observed that the VAS scores in the "Yes" group, that received the local steroid injection were consistently lower than those in the "No" group, who did not receive the injection; in the post operative follow up time intervals. However, this decrease in scores were not statistically significant.

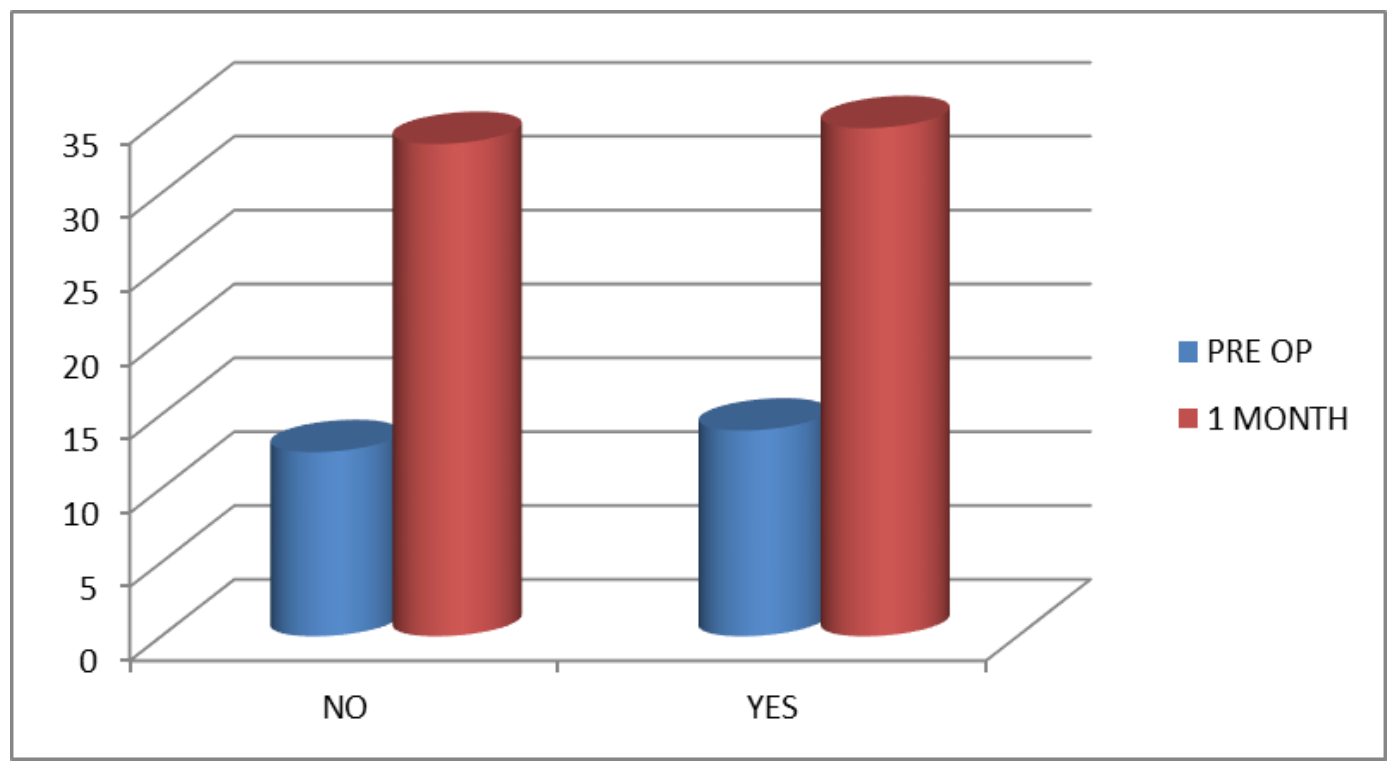

Graph 2: Comprison of Oxford score between the study groups at each time interval

This graph depicts the functional outcome (Oxford score) pre operatively and at 1 month, in the 2 groups. It is seen that both groups had a similar amount of significant improvement in functional scores at 1 month, and hence, the difference was insignificant.

Table 1: Pair-wise comparison of VAS between different time interval in each study group

\begin{tabular}{|c|c|c|c|c|c|c|c|}
\hline \multirow{2}{*}{ Injection } & \multirow{2}{*}{ (I) Time } & \multirow{2}{*}{ (J) Time } & \multirow{2}{*}{ Mean Difference (I-J) } & \multirow{2}{*}{ Std. Error } & \multirow{2}{*}{ p-value } & \multicolumn{2}{|c|}{ 95\% Confidence Interval for Difference } \\
\hline & & & & & & Lower Bound & Upper Bound \\
\hline \multirow{6}{*}{ No } & \multirow{3}{*}{ Pre - Op } & 4 Days & 4.87 & 0.26 & $<0.001^{*}$ & 4.08 & 5.65 \\
\hline & & 2 Weeks & 6.07 & 0.36 & $<0.001^{*}$ & 4.97 & 7.17 \\
\hline & & 1 Month & 5.07 & 0.40 & $<0.001^{*}$ & 3.85 & 6.28 \\
\hline & \multirow{2}{*}{4 Days } & 2 Weeks & 1.20 & 0.20 & $<0.001 *$ & 0.59 & 1.81 \\
\hline & & 1 Month & 0.20 & 0.36 & $1.00(\mathrm{NS})$ & -0.89 & 1.29 \\
\hline & 2 Weeks & 1 Month & -1.00 & 0.29 & $0.03 *$ & -1.90 & -0.10 \\
\hline \multirow{6}{*}{ Yes } & \multirow{3}{*}{ Pre - Op } & 4 Days & 4.87 & 0.32 & $<0.001^{*}$ & 3.88 & 5.85 \\
\hline & & 2 Weeks & 6.20 & 0.26 & $<0.001^{*}$ & 5.40 & 7.00 \\
\hline & & 1 Month & 5.67 & 0.33 & $<0.001 *$ & 4.64 & 6.69 \\
\hline & \multirow{2}{*}{4 Days } & 2 Weeks & 1.33 & 0.29 & $0.002 *$ & 0.45 & 2.22 \\
\hline & & 1 Month & 0.80 & 0.28 & $0.08(\mathrm{NS})$ & -0.06 & 1.66 \\
\hline & 2 Weeks & 1 Month & -0.53 & 0.19 & $0.09(\mathrm{NS})$ & -1.12 & 0.06 \\
\hline
\end{tabular}




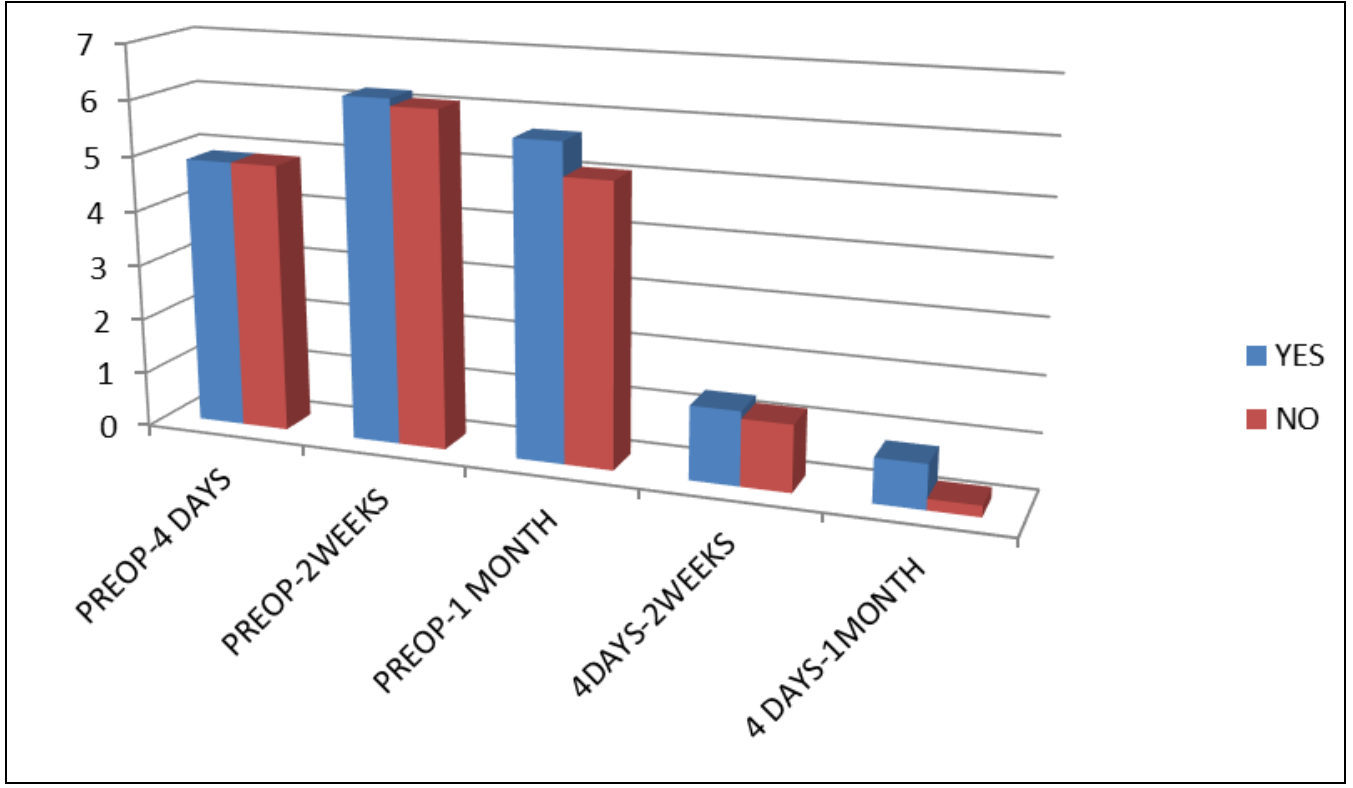

Graph 3: Pair-wise comparison of VAS between different time interval in each study group

This graph depicts the difference in VAS scores among different pairs of time intervals in both groups. It is evident that there was a greater improvement of pain scores up to 1 month in the study group that did receive the local corticosteroid injection.

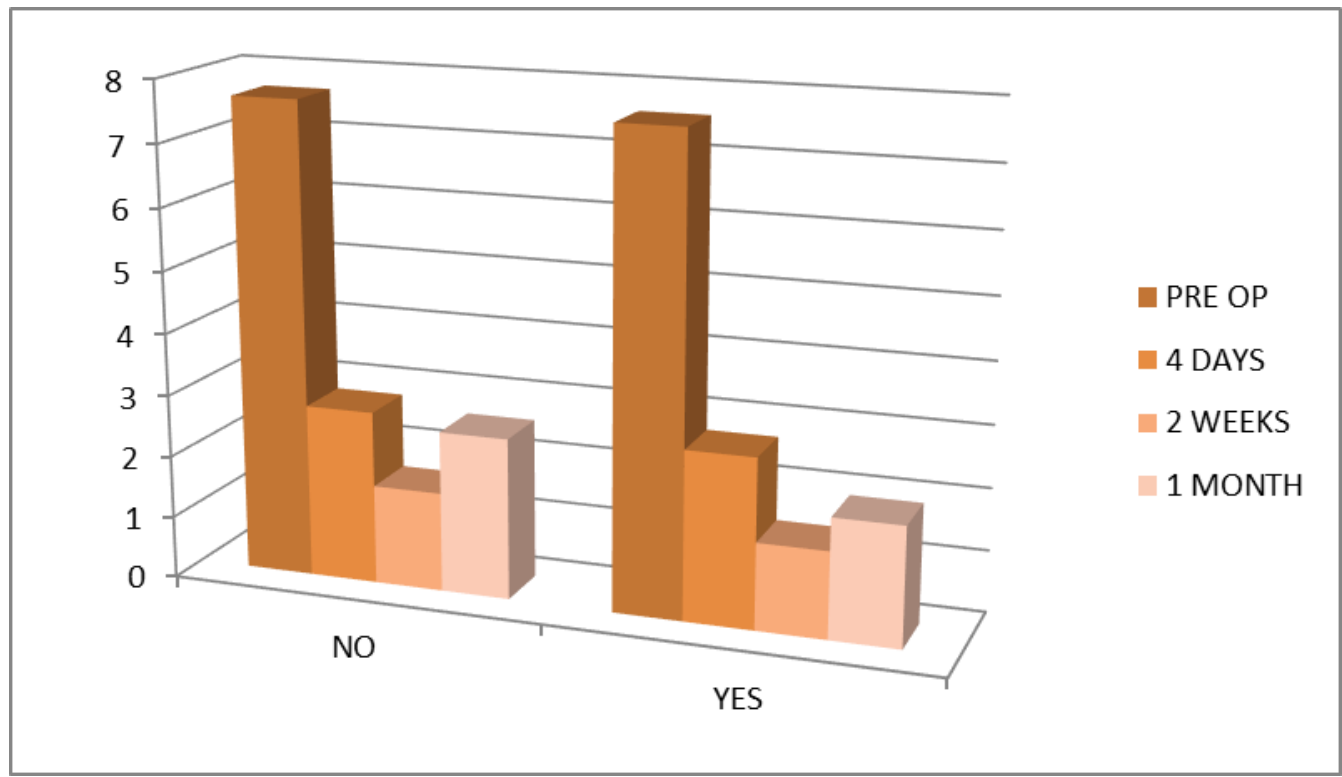

Graph 4: Comparison of VAS between different time intervals in each study group

In the graph above, the significant decreases in the VAS scores is noted in both groups upto 2 weeks. However, it is also observed that the pain scores increase at 1 month as compared to the 2 weeks interval, in both groups.
On comparing the scores from 2 weeks to 1 month, the pain scores had increased in both groups. However, this increase was insignificant in the "YES" group, whereas it was a statistically significant increase in the "NO" group. 


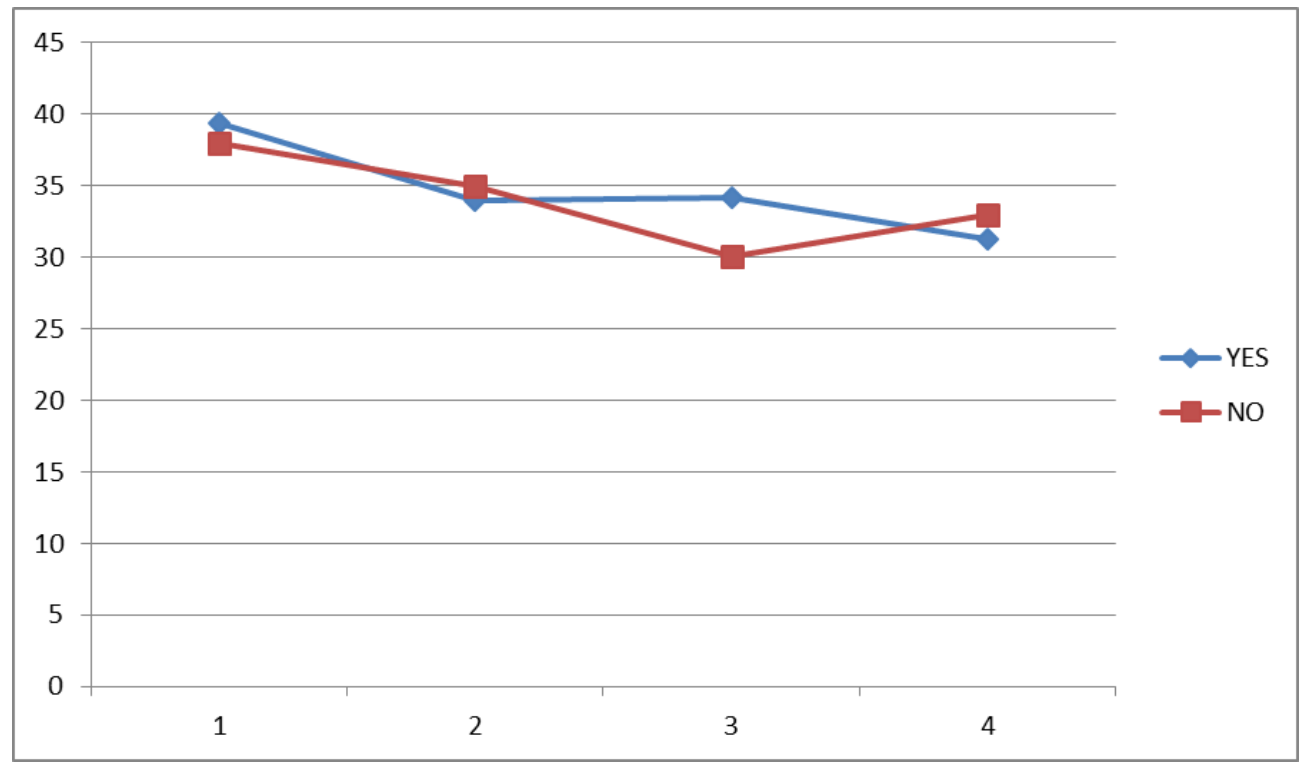

Graph 5: Comparison of Oxford score according to BMI in each study group

As above, when comparing oxford scores at 1month in patients with different categories of BMI, there was a varied decline in functional scores as the BMI increased, although this was observed to be statistically insignificant.

In either study group, there was no significant variation in VAS scores at different time intervals among the patients with different categories of BMI. Although there was a general trend of higher VAS scores with higher BMI categories, this was found to be insignificant in our study.

\section{Discussion}

Reports suggest that pes anserinus bursitis is far more common in overweight females, owing to the different angulation of the female knee, which puts more pressure on the area where the pes anserinus inserts. ${ }^{7}$ Asian authors evaluated 62 patients with knee osteoarthritis and verified the diagnosis of anserine bursitis, made by one rheumatologist, in $29(46 \%)$ patients, all females. The authors noted the frequent association between anserine bursitis and knee osteoarthritis. In another study ${ }^{[8]}$ of 68 patients with presumed osteoarthritis, 41 had anserine bursitis, of whom 38 were females and 37 were overweight.

In our study, the syndrome was found to occur exclusively in females. Moreover, most of the females were in the overweight and obese category. Their mean height was $152.04 \mathrm{~cm}$, mean weight was $71.94 \mathrm{~kg}$ and mean BMI was 30.92. These findings could lead to the proposed theory of varied angulation of the female knee, due to their wider pelvis, leading to increased pressures over the medial aspect of the knee. The increased weight could very well be an additional factor responsible for increased loads over the knee.

Alvarez et al. concluded that valgus knee deformity, alone or in association with collateral instability, seems to be a risk factor for PATB. Although pes anserine bursitis was believed to occur more frequently in the valgus knee, only $10 \%$ of our patients were diagnosed with valgus knees. In our study, $90 \%$ of the subjects had varus knees. This could be due to the far more increased prevalence of osteoarthritis knee with varus deformity compared to that with valgus. It could be postulated that the change in biomechanics due to the altered mechanical alignment, be it varus or valgus, could cause increased friction and eventually PATB. Another etiological factor in varus knees could be the prominent medial osteophytes present.

The high prevalence of $\mathrm{PAB}$ in patients with knee osteoarthritis, obesity, and angular deformity of the knee strongly suggests that mechanical derangement within the medial knee joint causes referred pain in the upper medial tibia. If the anserine bursa retains synovial fluid due to chronic inflammation from knee osteoarthritis, mechanical stress from walking may induce the rupture of anserine bursa. Reduction of the mechanical burden of the knee or increased muscle-support of the joint contributes to the prevention of recurrence of $\mathrm{PAB}$. Therefore, weight-reduction programs or quadriceps-strengthening exercises can reduce the incidence of this condition.

Our study revealed that the VAS scores of patients who received the injection were lesser than the scores of those who did not, at all post operative time intervals; the difference was statistically insignificant. These results indicate that there is a possible benefit in the post operative pain management protocol in patients undergoing TKR for osteoarthritis of the knee with associated pes anserine bursitis. The insignificant value could probably be due to the small sample size of our study, which is one of the limitations of this study.

The comparison of Oxford scores at the end of 1 month between the 2 study groups also did not reveal a statistically significant difference in our study. However, both groups had a statistically significant increase in the functional outcome post operatively at 1 month.

These results could also be an indication to the fact that the pain levels and loss of function that occur due to PAB syndrome in association with Grade 3 or 4 osteoarthritis of the knee can be effectively overcome by correction of the mechanical alignment and restoration of the normal anatomy of the knee, that is achieved with TKR. Another factor that compliments this recovery could be the controlled physical therapy that patients undergo post operatively for 1 month. The stretching and strengthening exercises included in the physical therapy regime could improve the kinematics of the muscle tendon units around the knee and hence lead to subsidence of the PAB syndrome.

Sarifakioglu et al. conducted a study comparing the effects of physical therapy and corticosteroid injection for pes anserine bursitis in patients with and without OA knee. In this study patients with PAB tended to have more severe pain, more altered functionality, and greater disability than those 
with OA but without PAB. Thus, both corticosteroid injection and physical therapy are effective methods of treatment for PAB. Injection therapy can be considered an effective, inexpensive and fast therapeutic method ${ }^{[9]}$.

In the post operative period, it was also noted that pain scores increased at 1 month compared to the scores at 2 weeks (Graph 4). This effect could be the result of the withdrawal of the post operative pain medications, aggressive physiotherapy, and a probable change in general perception and quality of life. This increase was found to be significantly lower in patients that received the local injection, indicating the possible prolonged beneficial effects of a local steroid injection for PAB syndrome.

The Table shows that, on comparison of VAS scores among different pairs of time intervals in between the study groups, the patients who received the injection had a better improvement in the time periods from "Pre-operative to 1 month" and also from "4 days to 1 month". This again is evidence of a beneficial effect of the local steroid injection for $\mathrm{PAB}$ with $\mathrm{OA}$.

On comparison of the VAS scores and the functional outcome scores among patient in different BMI categories, there was a general trend of decreased functional outcomes and increased pain scores among the patients with a higher BMI (Graph 5). Obesity is a well established etiological factor in the pathophysiology of osteoarthritis of the knee as well as pes anserine bursitis ${ }^{[7,8]}$. In this study, it is also seen that the patients with this syndrome tend to recover poorly when they are obese. Weight reduction and active lifestyle modifications are therefore a vital part of the rehabilitation program for these patients. Patients must understand the importance of the same before undergoing the surgical procedure so as to avoid failed expectations.

\section{Conclusion}

Local corticosteroid injections combined with TKR for PAB associated with osteoarthritis of the knee are safe but the efficacy of this treatment protocol could not be proved in our study. Our study did not reveal any statistically significant difference between the two treatment groups, and there was no consistent improvement in the functional recovery of patients undergoing the treatment protocol with local injections. The results were not statistically significant probably due to the small sample size of the study. Hence, further studies with larger sample size and greater post operative follow up period would be required to ascertain the beneficial effects of the combined therapy of local corticosteroid injections with TKR for PAB associated with osteoarthritis of the knee.

\section{References}

1. Neogi T. The epidemiology and impact of pain in osteoarthritis. Osteoarthritis Cartilage. 2013; 21(9):11451153. doi:10.1016/j.joca.2013.03.018

2. Saggini R, Di Stefano A, Dodaj I, Scarcello L, Bellomo RG. Pes Anserine Bursitis in Symptomatic Osteoarthritis Patients: A Mesotherapy Treatment Study. J Altern Complement Med. 2015; 21(8):480-484. doi:10.1089/acm.2015.0007

3. Rennie WJ, Saifuddin A. Pes anserine bursitis: Incidence in symptomatic knees and clinical presentation. Skeletal Radiol. 2005; 34:395-8.

4. Daniels EW, Cole D, Jacobs B, Phillips SF. Existing Evidence on Ultrasound-Guided Injections in Sports Medicine. Orthop J Sports Med. 2018;
6(2):2325967118756576. Published 2018 Feb 22. doi:10.1177/2325967118756576

5. Uson J, Aguado P, Bernad $\mathrm{M}$ et al. Pes anserinus tendino-bursitis: what are we talking about? Scand J Rheumatol, 2000; 29:184-186.

6. Rennie WJ, Saifuddin A. Pes anserine bursitis: Incidence in symptomatic knees and clinical presentation. Skeletal Radiol 2005; 34:395-8

7. Alvarez-Nemegyei J, Peláez-Ballestas I, Goñi M et al. Prevalence of rheumatic regional pain syndromes in Latin-American indigenous groups: a census study based on COPCORD methodology and syndrome-specific diagnostic criteria. Clin. Rheumatol. 2016; 35(1):63-70.

8. Larsson LG, Baum J. The syndrome of anserine bursitis: an overlooked diagnosis. Arthritis Rheum. 1985; 28:1062-1065.

9. Sarifakioglu B, Afsar SI, Yalbuzdag SA, Ustaömer K, Bayramoğlu M. Comparison of the efficacy of physical therapy and corticosteroid injection in the treatment of pes anserine tendino-bursitis. J Phys Ther Sci. 2016; 28(7):1993-1997. doi:10.1589/jpts.28.1993 